IFIC/95-24

\title{
Searching for $B_{c}$ mesons in the ATLAS experiment at LHC*
}

\author{
F. Albiol ${ }^{a, b \dagger}$, R. Pérez Ochoa ${ }^{a, b \ddagger}$, M. A. Sanchis-Lozano ${ }^{a, c \S}$ and J.A. Valls ${ }^{a, b \uparrow}$ \\ (a) Instituto de Física Corpuscular (IFIC) Centro Mixto Universidad de Valencia-CSIC \\ (b) Departamento de Física Atómica, Molecular y Nuclear \\ (c) Departamento de Física Teórica \\ Dr. Moliner 50, E-46100 Burjassot, Valencia (Spain)
}

October 1, 2018

\begin{abstract}
We discuss the feasibility of the observation of the signal from $B_{c}$ mesons in the ATLAS experiment of the LHC collider at a luminosity of $\approx 10^{33} \mathrm{~cm}^{-2} \mathrm{~s}^{-1}$. In particular we address the decay mode $B_{c} \rightarrow J / \psi \pi$ followed by the leptonic decay $J / \psi \rightarrow \mu^{+} \mu^{-}$, which should permit an accurate measurement of the $B_{c}$ mass. We performed a Monte Carlo study of the signal and background concluding that a precision of 40 $\mathrm{MeV}$ for the $B_{c}$ mass could be achieved after one year of running.
\end{abstract}

\section{Introduction}

There is a general consensus in the scientific community [1] that the scope of a future highluminosity, high-energy hadron collider like LHC should not be restricted to the hunting of the standard model Higgs and its extensions, or the search for supersymmetry. Other topics requiring lower luminosities like top and beauty physics deserve in their own right a close attention.

In this paper we shall not dwell on general aspects of $B$ physics already treated in detail in references [2], but rather we shall focus more specifically on the observation of $B_{c}(\bar{b} c)$ mesons. With regard to a general description of the ATLAS detector, technical details and foreseen performances we refer the reader to the Technical Proposal of the ATLAS Collaboration [3] and references therein. Finally let us observe that the aim of this paper is to extend and update our previous preliminary work on the detection of $B_{c}$ mesons presented as an ATLAS internal note [4].

From the theoretical point of view, $B_{c}$ mesons exhibit some unique features making them especially suitable for the study of the strong interaction dynamics in hadrons.

\footnotetext{
*Work partially supported by CICYT under contract AEN 93-0234 and by IVEI.

${ }^{\dagger}$ Now at the Liverpool University, P.O. Box 147, L693 BX, UK

${ }^{\ddagger}$ Now at CERN, PPE division, CH-1211 Geneva 23

$\S$ E-mail: mas@evalvx.ific.uv.es

๑Now at Fermilab, P.O. Box 500 Batavia, Illinois 60510
} 
First, $B_{c}$ states occupy an interpolating position in hadronic spectroscopy between charmonium and bottomonium resonances [5]. QCD-inspired theories like potential models can be submitted to a close scrutiny in such intermediate mass region, besides with different constituent quark masses. Moreover, low-energy effective theories like nonrelativistic QCD, which can be formulated on the lattice, may be applied as well either to heavy quarkonium formation or decay [6].

Furthermore, in contrast to singly heavy hadrons $\left(D, B, \Lambda_{c}, \Lambda_{b}, \ldots\right)$ both constituent quarks can undergo a weak decay, permitting a test of the "spectator" behaviour. (Let us note, nevertheless, that a simple-minded spectator model would not be valid since both heavy masses are involved in the hadron dynamics, even as an asymptotic limit.)

\section{$2 \quad B_{c}$ signal}

At the center-of-mass energy $\sqrt{s}=14 \mathrm{TeV}$, the cross-section for beauty production is assumed to be $500 \mu \mathrm{b}$ leading to $5 \times 10^{12} b \bar{b}$ pairs per year-run $\left(10^{7} \mathrm{~s}\right)$ at a luminosity of $\mathcal{L} \approx \infty^{\ni \ni} \mathrm{cm}^{-2} \mathrm{~s}^{-1}$, corresponding to an integrated luminosity of $\sim 10 \mathrm{fb}^{-1}$. The number of bottom pairs reduces, however, to $2.3 \times 10^{10}$ by requiring events with a triggering muon coming from either a $b$ or a $\bar{b}$ under the kinematic cuts $p_{\perp}>6 \mathrm{GeV} / \mathrm{c}$ and $|\eta|<2.2$ [3].

On the other hand, assuming that the $b$-quark fragmentation yields a $B_{c}$ or a $B_{c}^{*}$ with probability of the order of $10^{-3}[7]$, the yield of $B_{c}$ mesons (not yet triggered) per year of running would be roughly $\simeq 10^{10}$.

\section{$B_{c} \rightarrow J / \psi \pi$ channel}

This exclusive channel followed by the leptonic decay of the $J / \psi$ resonance into a pair of oppositely charged muons offers several important advantages. First of all, it allows for the mass reconstruction of the $B_{c}$ meson. Observe also that anyone of the two muons can trigger the decay. Besides, it is very clean topologically with a common secondary vertex for all three charged particles, two of them (the muons) with the additional constraint of their invariant mass compatible with a $J / \psi$. Furthermore, the expected branching fraction is not too small, about $0.2 \%$ [8], which combined with the branching fraction of the leptonic decay of the resonance $B R\left(J / \psi \rightarrow \mu^{+} \mu^{-}\right) \simeq 6 \%$, yields an overall branching fraction for the signal of $10^{-4}$. Thus, the number of such events turns out to be $\simeq 10^{6}$ per year of running.

\section{$B_{c} \rightarrow J / \psi \mu^{+} \nu_{\mu}$ channel}

In spite of the fact that this channel does not permit the measurement of the $B_{c}$ mass, its signature would be quite clean experimentally when the $J / \psi$ decays into a pair of muons, providing a three muon vertex. The overall branching fraction is then $\simeq 10^{-3}$ [9]. We leave the analysis and physical interest of this decay mode to a separate publication [10].

\footnotetext{
${ }^{1}$ The transverse momentum $p_{\perp}$ is measured with respect to the beamline and the pseudorapidity is defined as $\eta=-\ln \tan \theta / 2$ where $\theta$ is the polar angle
} 


\section{Detection efficiencies and background}

A study has been performed in order to estimate the signal detection efficiency and background for the $B_{c} \rightarrow J / \psi\left(\rightarrow \mu^{+} \mu^{-}\right) \pi$ channel. The Monte Carlo employed for the signal simulation corresponds to a sample of $B_{c} \rightarrow J / \psi \pi$ events 1 while for the background we have used a sample of inclusive $b$ muon decays generated in all the cases with PYTHIA 5.7 .

A full simulation of the ATLAS detector using the GEANT Monte Carlo program was performed to study in detail the reconstruction of $B$ events, parametrizing the detector effects through smearing routines [11]. Consequently, our signal and background analysis were performed using a particle-level simulation with parametrized momentum and impact parameter resolutions [3].

In this paper, we shall consider two types of background: [

a) Combinatorial background due to muons from semileptonic decays of $b \bar{b}$ pairs produced at the main interaction. Cascade contributions such as $b \rightarrow c \rightarrow \mu$ are included as well for random combinations with any other muon in the same event.

b) Contamination from prompt $J / \psi$ 's in combination with another charged hadron (interpreted as a pion) from the main vertex. (In fact data released by Tevatron on the $J / \psi$ yield point out a production rate quite larger than initially expected [12].) Incorrect tracking may give rise to the reconstruction of a (fake) secondary vertex, becoming a potential source of a large amount of background.

In a first step, we imposed the following cuts on events based on kinematic constraints:

- $p_{\perp \min }($ trig. $\mu)=6 \mathrm{GeV} / \mathrm{c} ; \mid \eta_{\max }($ trig. $\mu) \mid=2.2$

- $p_{\perp \min }(\mu)=3 \mathrm{GeV} / \mathrm{c} ;\left|\eta_{\max }(\mu)\right|=2.5$

- $p_{\perp \min }(\pi)=1 \mathrm{GeV} / \mathrm{c} ;\left|\eta_{\max }(\pi)\right|=2.5$

- $M_{\mu^{+} \mu^{-}}=M_{J / \psi} \pm 50 \mathrm{MeV}$

The two first cuts correspond to the requirement of the 1st-level $B$ physics trigger leading in our case to an efficiency of $\sim 15 \%$ in triggering one of the two muons from the $J / \psi$. We next take into account the detection efficiency for the signal after applying the rest of $p_{\perp}$ and $\eta$ cuts which turns out to be $\sim 21 \%$. Setting the efficiency for muon identification as $80 \%$ and the track reconstruction as $95 \%$ [3] we get a combined detection efficiency of $\sim 2 \%$ leading to an observable signal of about 20,000 events per year of running.

The last of the cuts described above constrains the two muons invariant mass to be compatible (within two standard deviations [3]) with the nominal $J / \psi$ mass, thus drastically reducing random combinations. However, background of class $b$ ) can potentially pass all the kinematic cuts by a large amount, so another type of rejection is required.

\footnotetext{
${ }^{2}$ We are here interested in the efficiency of the signal rather than in absolute production rates for which we made a rough estimate in the previous section according to fragmentation of $b$ quarks into $B_{c}$ mesons

${ }^{3}$ Muons coming from semileptonic decays of long-lived particles such as pions or kaons contribute in a negligible amount to trigger rates. On the other hand $B$ decays into $J / \psi$ and a charged particle would give an invariant mass quite below the $B_{c}$ mass so they are of no concern
} 
To this end, we adapted to our needs the vertex reconstruction (i.e. vertex finding and fitting) routines of the LEP experiment DELPHI at CERN [13] 1. The vertex fitting algorithm provides as output the coordinates of the secondary vertex, the track momenta re-evaluated with the vertex constraint and the goodness of the fit by means of the total $\chi^{2}$ as well as the contribution of each single track to it. In particular, we employed for background rejection the three spatial coordinates and the $\chi^{2}$ per degree of freedom for each fitted secondary vertex formed by the two muons and the charged hadron (assumed to be a pion) satisfying the above kinematic constraints. The distance between the reconstructed vertex and the primary $(p p)$ interaction point was thereby determined. We shall refer to it as the decay length even for background events of class b).

Hence, candidate (either signal or background) events were required to pass the following extra cuts:

- total $\chi^{2}<\chi_{0}^{2}$

- $\chi_{i}^{2}<\frac{\chi_{0}^{2}}{3}$ for each single track- $i$

- decay length larger than $L_{0}$

where $\chi_{0}^{2}, L_{0}$ have to be optimized to remove the background as much as possible but with a good acceptance for the signal. In our analysis we found $\chi_{0}^{2}=8$ and $L_{0}=350 \mu \mathrm{m}$.

In figure 1 the effects of these cuts on signal and background events are shown. Let us remark that with this selected range of $\chi^{2}$ and decay length, the background of class $a$ ) falls to the $4.5 \%$ (with respect to the background after the kinematic cuts), the background of class $b$ ) is completely removed, whereas the acceptance for the signal turns out to be $46 \%$. The total rejection of the contamination from prompt $J / \psi$ 's can be quickly understood since actually they are produced (thus decaying) at the $p p$ interaction vertex itself. Those fake secondary vertices "reconstructed faraway" from the main vertex give rise to a quite large $\chi^{2}$ in the fit, so being removed by these combined cuts.

Figure 2 shows the reconstructed $\left(\mu^{+} \mu^{-}\right)_{J / \psi} \pi$ mass distribution for the expected signal above the surviving background once all the cuts have been applied, for an integrated luminosity of $10 \mathrm{fb}^{-1}$.

\section{Summary}

We have found that the self-triggering weak decay $B_{c} \rightarrow J / \psi \pi$, followed by the leptonic decay of the $J / \psi$ into two muons, could be clearly observed in the ATLAS detector at LHC. Under rather conservative assumptions, a total number of $\approx 10,000$ signal events could be fully reconstructed after one year run, corresponding to $10 \mathrm{fb}^{-1}$ at "low" luminosity $\left(\approx 10^{33}\right.$ $\mathrm{cm}^{-2} \mathrm{~s}^{-1}$ ). This represents a signal to background ratio of about 0.5 with a statistical significance of $\approx 20$ standard deviations above a nearby almost flat background. The foreseen mass resolution of the $B_{c}$ meson is about $40 \mathrm{MeV}$.

\footnotetext{
${ }^{4}$ We are indebted to E. Cortina for technical advice in this task

${ }^{5}$ No candidate event was found in a sample corresponding to 100,000 prompt $J / \psi$ 's
} 


\section{Acknowledgments}

We are indebted to P. Eerola and N. Ellis and the B-Physics Group of the ATLAS Collaboration for their interest and advice. One of us (M.A.S.L.) thanks the Vice-Rectorado de Investigación de la Universitat de València for partial support.

\section{References}

[1] G. Carboni et al, Proceedings of the ECFA Large Hadron Collider Workshop, Aachen (1990)

[2] CERN preprint CERN/LHCC/93-53 (1993); ATLAS internal note PHYSICS-NO-041 (1994); P. Eerola et al., Nucl. Instr. and Meth. A351 (1994) 84

[3] Technical Proposal of the ATLAS Collaboration, CERN/LHCC 94-43

[4] ATLAS internal note, PHYS-NO-058 (1994)

[5] E. Bagan et al, Z. Phys. C64 (1994) 57

[6] G.T. Bodwin, E. Braaten and G.P. Lepage, Phys. Rev. D51 (1995) 1125

[7] K. Cheung and T.C. Yuan, hep-ph/9502250 ; K. Cheung and T.C. Yuan, Phys. Lett. B325 (1994) ; E. Braaten, K. Cheung and T.C. Yuan, Phys. Rev. D48 (1993) 5049 ; D48 (1993) R5049 ; K. Cheung, Phys. Rev. Lett. 71 (1993) 3413

[8] M. Lusignoli and M. Masetti, Z. Phys. C51 (1991) 549

[9] M.A. Sanchis-Lozano, Nuc. Phys. B440 (1995) 251

[10] M. Galdón and M.A. Sanchis-Lozano, IFIC/95-31

[11] We thank P. Eerola for providing us the smearing routines

[12] M. Cacciari and M. Greco, Phys. Rev. Lett. 73 (1994) 1586; E. Braaten, M.A. Doncheski, S. Fleming and M.L. Mangano, Phys. Lett. B333 (1994) 548

[13] P. Billoir and S. Qian, Nucl. Instr. and Meth. A311 (1992) 139 

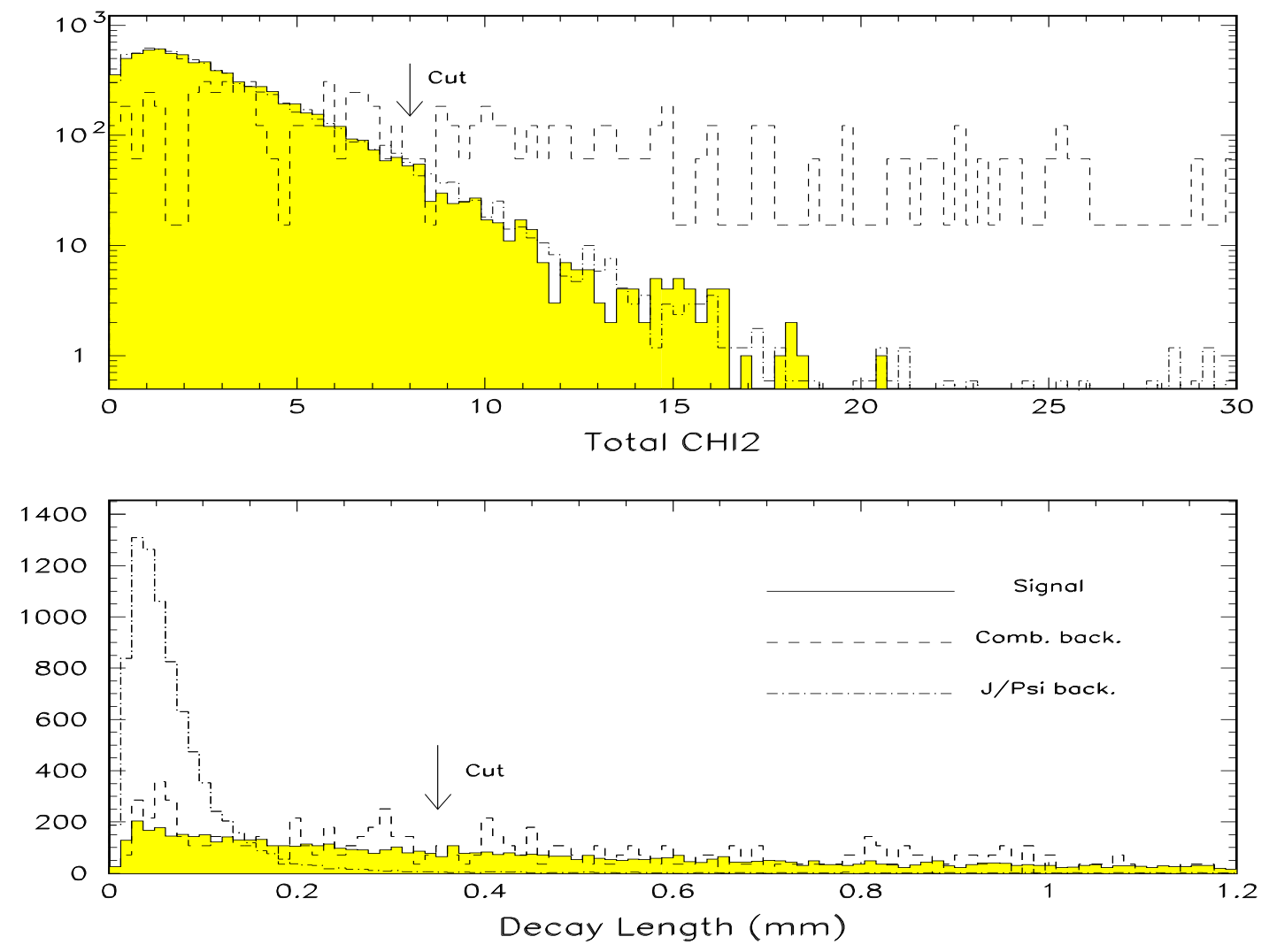

Figure 1: Effect of the vertex reconstruction cuts on the signal (solid line) and background of class $a$ ) (dashed line) and class $b$ ) (dot-dashed line), separately. The cut on the $\chi^{2}$ was set equal to 8 and the decay length cut was $350 \mu \mathrm{m}$. All three samples are normalized to the same number of events. 


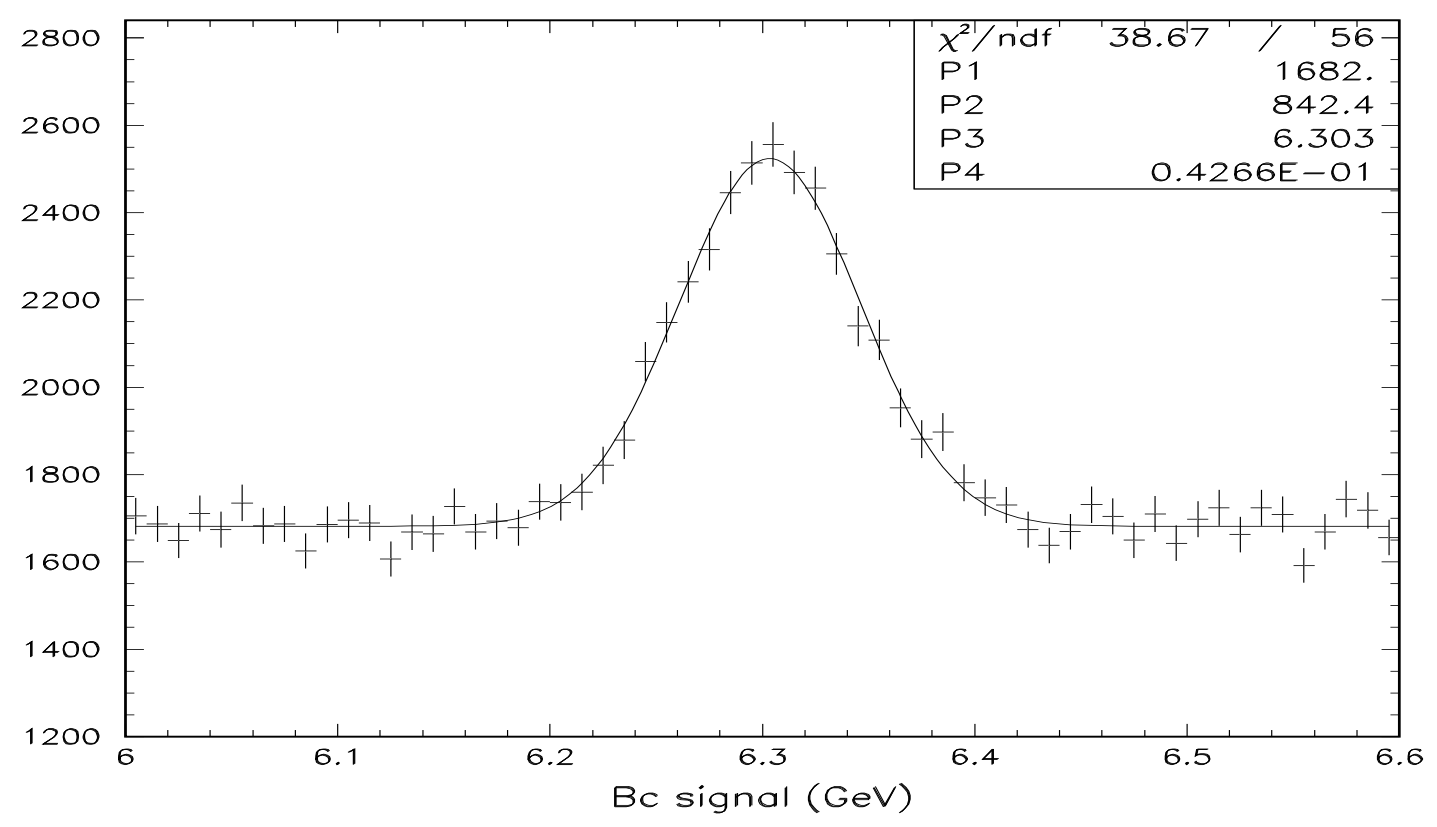

Figure 2: Reconstructed $\left(\mu^{+} \mu^{-}\right)_{J / \psi} \pi$ mass distribution after cuts. The nominal value for the $B_{c}$ was set equal to $6.3 \mathrm{GeV}$. The solid line corresponds to a linear+Gaussian fit. 